Man and Nature

MAN AND NATURE

L'homme et la nature

L'HOMME ET LA NATURE

\title{
Deux confrontations du sauvage et du civilisé : les Dialogues de Lahontan et le Supplément au voyage de Bougainville de Diderot
}

\section{Henri Coulet}

Volume 9, 1990

URI : https://id.erudit.org/iderudit/1012614ar

DOI : https://doi.org/10.7202/1012614ar

Aller au sommaire du numéro

Éditeur(s)

Canadian Society for Eighteenth-Century Studies / Société canadienne d'étude du dix-huitième siècle

ISSN

0824-3298 (imprimé)

1927-8810 (numérique)

Découvrir la revue

Citer cet article

Coulet, H. (1990). Deux confrontations du sauvage et du civilisé : les Dialogues de Lahontan et le Supplément au voyage de Bougainville de Diderot. Man and Nature / L'homme et la nature, 9, 119-132. https://doi.org/10.7202/1012614ar

Copyright (c) Canadian Society for Eighteenth-Century Studies / Sociéte canadienne d'étude du dix-huitième siècle, 1990
Ce document est protégé par la loi sur le droit d'auteur. L'utilisation des services d'Érudit (y compris la reproduction) est assujettie à sa politique d'utilisation que vous pouvez consulter en ligne.

https://apropos.erudit.org/fr/usagers/politique-dutilisation/ 


\section{Deux confrontations du sauvage et du civilisé: les Dialogues de Lahontan et le Supplément au voyage de Bougainville de Diderot}

Entre 1700 et 1790, nombreux sont les textes où la critique de la civilisation européenne est faite par un sauvage américain, africain ou océanien. Pourquoi extraire de cet ensemble les Dialogues de Lahontan (Supplément aux voyages du baron de Lahontan où l'on trouve des Dialogues curieux entre l'auteur et un sauvage de bon sens qui a voyagé, 1703) et le Supplément au voyage de Bougainville de Diderot (écrit en 1771, corrigé en 1773 et 1778-1779, publié pour la première fois en 1796) ? Mis à part le thème général qu'ils ont en commun, leurs différences sautent aux yeux: les Dialogues ont pour auteur un aventurier des dernières années du XVIIe siècle; le Supplément, un écrivain professionnel, un philosophe de l'époque des Lumières; dans le premier écrit, le sauvage est un Huron, dans l'autre un Tahitien; chez les Hurons, l'existence est rude, la chasse n'assure pas toujours la subsistance de tous, la guerre sévit entre les peuples; chez les Tahitiens, l'existence est douce, la nature généreuse, le plaisir partagé par tous. Mais ces deux textes se ressemblent par la façon dont le civilisé y prend conscience de lui-même en faisant parler le sauvage. Sauf Voltaire, qui dans Candide laisse les sauvages à leur sauvagerie, et dans l'Ingénu montre comment un Huron, dont l'origine n'est d'ailleurs pas sauvage, s'adapte à la civilisation, la plupart des écrivains qui ont représenté dans un récit, dans des lettres ou dans des dialogues la confrontation du sauvage et de l'Européen se sont servis d'un procédé d'exposition à la mode pour faire une critique piquante des moeurs et des croyances de leurs contemporains. Ni Bricaire de la Dixmerie (Le Sauvage de Tahiti aux Français, 1770), ni Maubert de Gouvest (Lettres iroquoises, 1752), pour citer deux auteurs dont les sauvages viennent des mêmes pays que ceux de Diderot et de Lahontan, ne se sont un instant demandé si le sauvage pouvait juger la civilisation, si le civilisé pouvait faire parler le sauvage et à quelles conditions le civilisé pouvait trouver un sens pour lui dans l'existence 
du sauvage. Au contraire, Lahontan et Diderot se sont posé la question, qu'on ne peut éluder sans réduire la critique de la civilisation à une satire superficielle.

L'oeuvre de Lahontan a été utilisée partie directement, partie par l'intermédiaire de l'Histoire critica philosophiae de Brucker, dans l'article de l'abbé Pestré: 'Philosophie des Canadiens' de l'Encyclopédie. ${ }^{1}$

Diderot avait certainement lu les Mémoires de l'Amérique septentrionale, qu'il cite en 1761 ou 1762 dans la 40ème pensée de l'Addition aux pensées philosophiques; il avait dû lire aussi les Nouveaux voyages de Mr. le baron de Lahontan dont les Mémoires étaient la suite et le second tome. Quant aux Dialogues, qui avaient paru comme troisième tome, mais séparément, il n'en fait aucune mention, mais il semble bien qu'il leur doivel'idée même du dialogue entre Orou et l'aumônier. Le titre de son oeuvre rappelle celui des Dialogues: Supplément aux voyages $d u$ baron de Lahontan. ${ }^{2}$ Dans le dialogue, l'attitude de l'Européen, incapable de répondre solidement aux accusations du sauvage et presque forcé de lui donner raison, est cellede Lahontan en face d'Adario. Analogieplus importante, ledialogue a lieu dans le pays du sauvage; la plupart, sinon la totalité, des oeuvres dans lesquelles un étranger, sauvage ou non, critique la société européenne, montrent cet étranger en Europe, où il s'étonne de ce qu'il voit et étonne réciproquement les Européens. Tout change si l'étranger est chez lui, où les Européens sont venus, comme l'imaginent Lahontan et Diderot après lui: les civilisés ne sont plus des objets à regarder, bizarres, ridicules ou vicieux, ils sont des envahisseurs, des agresseurs; la situation dans laquelle se trouvent les interlocuteurs n'est pas une situation d'altérité, mais d'hostilité, même si les Européens ne croient pas venir en ennemis, même si le rapport personnel d'un interlocuteur à l'autre, d'Orou à l'aumônier, d'Adario à Lahontan est celui de l'hospitalité et de la fraternité humaine. D'emblée la civilisation est porteuse de violence et d'aliénation. Le civilisé projette son propre malaise sur autrui et le concrétise dans son rapport avec le sauvage.

Les deux oeuvres se rattachent à des écrits documentaires, les Nouveaux Voyages et les Mémoires pour l'une, le Voyage de Bougainville pour l'autre. Elles s'y rattachent, mais pour s'en détacher: elles sont orientées vers un autre but que celui d'instruire le lecteur sur une réalité exotique. Cette disjonction et cette réorientation sont évidentes pour le Supplément de Diderot: Diderot est parti d'un compte rendu du Voyage de Bougainville qu'il avait rédigé pour Grimm et qu'il n'avait pas publié, il y a ajouté le dialogue-cadre entre $\mathrm{A}$ et $\mathrm{B}$, et il a explicitement réuni la nouvelle oeuvre à deux contes, Madame de la Carlière et Ceci n'est pas un conte, avec lesquels elle forme un triptyque. L'autonomie des Dialogues de Lahontan par rapport aux Nouveaux Voyages et aux Mémoires est moins apparente. La plupart des critiques, 
ayant bien vu que l'image de la vie sauvage y était déformée pour faciliter la démonstration, ont jugé de mauvaise foi le rôle ridicule $\mathrm{d}^{\prime}$ interlocuteur que s'attribuait Lahontan et pensé que le dialogue était de pure imagination, et parce qu'ils tenaient les Dialogues pour une imposture, ils ont accusé de fausseté les Nouveaux Voyages et les Mémoires également; mais à l'inverse, ils ont recherché dans les Dialogues des renseignements ethnologiques comme leur en donnaient les Nouveaux Voyages et les Mémoires; ces deux lectures opposées se réunissant, les Dialogues sont souvent considérés à la fois comme un document authentique sur les Amérindiens et comme une fable satirique, assemblage informe d'expérience vécue et d'invention polémique. Certes, Lahontan part, pour écrire les Dialogues, des souvenirs authentiques qu'il a du Canada, mais il en fait tout autre chose que dans les Nouveaux Voyages et dans les Mémoires. Il faut restituer à chacun des trois textes sa finalité propre: les Nouveaux Voyages, que les lettres qui les composent aient été réellement envoyées à un correspondant ou, comme il est plus probable, qu'elles aient été arrangées et ordonnées pour leur publication, racontent l'expérience personnelle de Lahontan au Canada, ce qu'il a appris, ce qu'il a compris, donc la compétence qu'il a acquise et le dépit que lui ont causé l'incapacité des plus hauts responsables et la malveillance des ministres: description des réalités américaines, histoire des événements, histoire personnelle convergent vers une conclusion implicite à l'adresse des puissances gouvernementales, par dessus la tête du destinataire supposé, et qu'on pourrait formuler ainsi: 'je sais ce dont je parle, j'ai fait mes preuves, écoutez-moi au lieu de me repousser.' $C^{\prime}$ est bien à une telle conclusion que tend la démolition du discours explicatif qu'ont bien analysée Claude Rigault et Réal Ouellet. ${ }^{3}$ L'his- $^{\prime}$ toire officielle, celle que croient écrire par leurs décisions et par leurs actions les détenteurs du pouvoir, est une illusion; à l'épreuve, les desseins des puissants sont inefficaces et absurdes, le hasard seul détermine les événements; mais cette absurdité des calculs et ce rôle déterminant du hasard s'expliquent par une cause aisément identifiable: l'ignorance de la réalité américaine. Lahontan, qui agit quotidiennement sur le terrain et parmi les habitants du pays, récrit l'histoire vraie, dans son incohérence dont il nous donne la clef. Les Mémoires s'inscrivent tout naturellement à la suite de cette histoire récrite et expliquée, ils font aussi méthodiquement que possible le tableau de la réalité qu'il faut connaître si l'on veut agir efficacement en Amérique du Nord; les chapitres sur la religion, sur l'amour et le mariage chez les sauvages, ne sont pas des pamphlets philosophiques, ils exposent, comme les chapitres sur la géographie, sur la faune, sur le commerce et sur le gouvernement, les données dont toute action devra tenir 
compte. Et si, au passage, telle qu'elle est décrite par Lahontan, la croyance des sauvages en Dieu ressemble étrangement au panthéisme spinoziste que professent certains textes clandestins antireligieux, ce n'est pas par une intention malicieuse de Lahontan, c'est parce qu'il veut traduire dans des termes compréhensibles aux Européens une mentalité étrangère; la déformation ici n'est pas polémique, elle est destinée à faciliter la compréhension puisque, Lahontan nous le dit, son correspondant lui avait reproché d'avoir rapporté les paroles du chef sauvage Grangula dans le vrai style des sauvages. En somme, dans ces deux oeuvres complémentaires, Lahontan s'exprime en Européen conquérant et colonisateur (un détail caractéristique: le Petit dictionnaire de la langue des sauvages mis à la fin des Mémoires est un lexique français-huron, et non huron-français), mais en colonisateur intelligent, qui sait quand et comment il faut négocier, qui il faut aider, qui il faut craindre, qui il faut réprimer, quand il faut menacer de la force et quand il faut s'en servir, etc. Et, seion lui, l'une des plus graves erreurs commises par la France au Canada est d'avoir laissé s'implanter un pouvoir clérical dont l'esprit des sauvages ne peut pas concevoir les dogmes, qui fausse toute action politique et interdit aux Français (différents en cela des Anglais) de voir les sauvages tels qu'ils sont et de les traiter selon leur véritable nature. Les Dialogues, en revanche, ne sont plus l'oeuvre de l'officier colonial mais du penseur, du philosophe critique qui se propose de comprendre sa propre identité de civilisé mal à son aise dans la civilisation, et non pas d'agir sur une réalité étrangère à la plupart de ses compatriotes. La réalité américaine est alors déformée, au bénéfice de la réflexion philosophique et de la satire, autant que l'est la réalité tahitienne dans le Supplément de Diderot.

Les deux écrivains ont suffisamment averti leurs lecteurs de cette déformation, les indices ne manquent pas qui invitent à une lecture ironique. Voici un exemple dans les Dialogues; c'est Adario le Huron qui oppose la solide santé des sauvages à la mollesse maladive des Européens: 'Déjà parmi nous on ne voit quasi jamais ni bossus, ni boiteux, ni nains, ni sourds, ni muets, ni aveugles de naissance, encore moins de borgnes; et quand ces derniers viennent au monde, $c^{\prime}$ est un présage assuré de malheur à la nation, comme nous l'avons souvent observé. Tout borgne n'eut jamais d'esprit ni de droiture de coeur; au reste malicieux, paillard et paresseux au dernier point; plus poltron que le lièvre, n'allant jamais à la chasse de crainte de crever son oeil unique à quelque branche d'arbre [etc.]' (édition originale, p.78). ${ }^{4}$ Pourquoi cette attaque insistante, et d'une éclatante mauvaise foi, contre les borgnes, que les Mémoires se contentaient de citer parmi les autres infirmes (Mémoires, p.94)? A la différence des éditeurs modernes, les 
lecteurs de 1703 ne s'y seront pas trompés: Lahontan se vengeait d'un borgne qu'il n'aimait pas, le secrétaire d'Etat à la marine Pontchartrain, celui que Saint-Simon, qui le détestait aussi, appelait 'le cyclope' et dont il a laissé dans sa chronique de 1711 (Mémoires, éd. Yves Coirault, t.IV, p.250-251) un portrait hideux au moral comme au physique. L'incongruité de cette bouffonnerie satirique dans les Dialogues nous avertit que nous sommes loin de l'ethnologie. ${ }^{5}$

Mais l'indice le plus clair et le plus répété de l'intention ludique et polémique des Dialogues est le rôle que Lahontan s'y attribue: c'est celui de l'interlocuteur sot et naif, celui du Jésuite dans les Lettres provinciales et du père Canaye dans la Conversation du maréchal d'Hocquincourt, de Saint- Evremond. Le lecteur ne peut pas ne pas donner raison à Adario contre son interlocuteur, mais il ne peut non plus croire que Lahontan soit réellement aussi maladroit et aussi borné qu'il affecte de l'être: 'Je ne saurais nier que...,' 'Il est bien vrai ...,' 'J'avoue que tu as raison ...,' 'Je te renvoie aux Jésuites ...'; ou encore, à propos des Anglais qui vont au ciel sans avoir besoin du pape: 'Le Fils de Dieu veut les sauver tous par son sang et ses mérites. Or, s'il le veut, il faut que cela soit ainsi. Ainsi, tu vois bien qu'ils sont plus heureux que les Français, dont ce Dieu exige de bonnes oeuvres qu'ils ne font guère'; ou bien à propos des juges qui en France sont tous justes et désintéressés et ont fait perdre à Lahontan ses procès contre des adversaires plus riches et plus influents: 'Je serais bien fâché de croire qu'ils les ont mal jugés [. . .]. Ce sont les lois qui m'ont jugé et les lois sont justes et raisonnables. Je croyais avoir raison parce que je ne les avais pas bien étudiées.' On pourrait citer vingt exemples de raisonnements boiteux, de recours risibles à l'autorité, $\mathrm{d}^{\prime}$ arguments qui prouvent le contraire de ce qu'ils devraient démontrer, de reproches de ne pas comprendre adressés au sauvage parce qu'on ne sait pas le convaincre. C'est déjà le ridicule qu'on trouvera dans l'aumônier du Supplément, mais Diderot ne se confondra pas avec l'aumônier. Adario s'étonne, et l'auteur vend la mèche, bien que le lecteur n'ait guère risqué d'être dupe: ' $C$ 'est quelque chose d'étrange que, depuis que nous parlons ensemble, tu ne me répondes que superficiellement sur toutes les objections que je $t^{\prime}$ ai faites. Je vois que tu cherches des détours et que tu t'éloignes toujours du sujet de mes questions. $^{\prime}$ Le sens du dialogue est à chercher dans cette complicité proposée au lecteur, dans l'ironie envers un entretien faussé.

L'ironie, ou, si l'on préfère un mot plus à la mode, la distanciation est plus subtilement produite dans le Supplément au voyage de Bougainville. Dès le titre, le lecteur peut s'interroger: Supplément au voyage de Bougainville ou dialogue entre A et B. H. Dieckmann, qui a donné en 1955 une édition critique de cette oeuvre, précédée d'une longue et riche introduction, pensait qu'au lieu de ou, Diderot aurait dû écrire et, le 
dialogue de $\mathrm{A}$ et $\mathrm{B}$ encadrant et enchaînant les scènes tahitiennes (adieux du vieillard et entretien de l'aumônier et d'Orou) qui constitueraient le Supplément proprement dit. Mais Diderot veut nous faire entendre que dialogue et scènes tahitiennes ne font qu'un, appartiennent au même registre d'expression, et constituent ensemble, inséparablement, le supplément au voyage de Bougainville. Dans le texte même, les indices de l'ironie abondent. Quand A objecte à B, qui oppose la jeunesse et la liberté du monde sauvage à la décrépitude du monde civilisé: 'Est-ce que vous donneriez dans la fable de Tahiti?,' B répond: 'Ce n'est point une fable; et vous n'auriez aucun doute sur la sincérité de Bougainville, si vous connaissiez le supplément de son voyage,' il allègue comme preuve de la vérité un récit entièrement imaginaire, qui ne peut offrir quelque vérité que si elle lui vient du Voyage de Bougainville lui-même: façon plaisante d'avouer que c'est le Supplément qui est une 'fable.' Dans la suite du texte, l'interlocuteur de l'aumônier est Orou, non Aotourou, dont B nous a dit qu'il aurait été incapable de concevoir et de faire comprendre à ses congénères les usages des civilisés; Orou, au contraire, se montre fort capable de les discuter avec autant d'énergie que d'intelligence. Au contact réel du civilisé et du sauvage, qui selon lui était dépourvu de sens, Diderot substitue un affrontement hautement significatif. Le véritable Aotourou qui, en vrai sauvage dépaysé, se jeta 'sur la première Européenne qui vint à sa rencontre' et 'se disposait très sérieusement à lui faire la politesse de Tahiti,' s'ennuya en France, 'il ne put jamais apprendre à parler notre langue', 'il ne cessait de soupirer après son pays' (p.463). Ces détails authentiques, Diderot ne les trouvait pas dans Bougainville, mais dans les témoignages de ceux qui avaient connu le sauvage à Paris. ${ }^{7}$ Bougainville, au contraire, peut-être pour prouver contre Rousseau que l'homme est naturellement fait pour la civilisation, prétendait qu'Aotourou avait été parfaitement à son aise dans la vie parisienne, savait se faire indiquer son chemin, achetait dans les boutiques sans se faire voler et allait même tout seul à l'Opéra. Diderot, en rétablissant ce qu'il pense être la vérité, met une distance infranchissable entre le sauvage réel, enfermé dans sa sauvagerie au milieu de la civilisation, et le sauvage imaginaire, esprit lucide et critique, orateur vigoureux et adroit. Enfin le Supplément est explicitement relié aux contes de Madame de la Carlière et de Ceci n'est pas un conte. Il serait trop long de démontrer ici l'unité de ces trois textes, notons seulement que la leçon des deux contes est qu'il faut être tolérant dans la société et ne pas traiter les faiblesses comme des crimes. Ne prenons donc pas pour une inconséquence ou un jeu gratuit le contraste entre la critique sévère de la civilisation qui est au coeur du Supplément et les conseils d'accommodement et de modération qui le terminent. 
Il y a un jeu dans le Supplément, mais il ne consiste pas à encadrer par des réflexions de morale sociale une fantaisie exotique sans rapport avec elles: ce jeu est d'inviter le lecteur à ne pas prendre à la lettre les entretiens tahitiens et à en chercher le sens dans les conseils terminaux de B. C'est un jeu, par exemple, que les adieux du vieillard; Diderot a dû s'en réjouir intérieurement, quand très peu de lecteurs pouvaient le discerner: cette annonce apocalyptique des malheurs qui attendent les sauvages envahis par les Européens, cette exhortation indignée à Bougainville de quitter Tahiti avant que le pire ne soit accompli, étaient, dans le compte rendu primitivement écrit pour Grimm en 1771, une prosopopée de Diderot lui-même: 'Ah! Monsieur de Bougainville, éloignez votre vaisseau de ces innocents et fortunés Tahitiens [etc]. ${ }^{8}{ }^{~} \mathrm{Ce}$ discours (remarquait $\mathrm{A}, \mathrm{p}$.472) me paraît véhément; mais à travers je ne sais quoi d'abrupt et de sauvage, il me semble retrouver des idées et des tournures européennes,' et l'explication apportée par B, comique et invraisemblable, devait éveiller les soupçons du plus innocent lecteur: 'Pensez donc que c'est une traduction du tahitien en espagnol, et de l'espagnol en français. Le vieillard s'était rendu, la nuit, chez cet Orou qu'il a interpellé, et dans la case duquel l'usage de la langue espagnole s'était conservé de temps immémorial. Orou avait écrit en espagnol la harangue du vieillard; et Bougainville en avait une copie à la main, tandis que le Tahitien la prononçait.' Tout cela est extravagant; Diderot se moque du lecteur, dans tout ce passage et dans bien d'autres, et l'oblige à ne pas s'en tenir à la surface du texte. Orou non plus ne s'exprime pas en sauvage: son discours paraît à $\mathrm{A}$ 'un peu modelé à l'européenne' et B lui répond, sans plus de commentaire: 'Je n'en doute pas.' La jeune Thia, la plus jeune fille d'Orou, réclame un enfant de l'étranger avec l'accent de Didon abandonnée par Enée, au livre IV de l'Enéide (vers 327-330): 'Saltem si qua mihi de te suscepta fuisset / Ante fugam suboles, si quis mihi parvulus aula / Luderet Aeneas . . . ' 'Etranger, honnête étranger, ne me rebute pas! rends-moi mère, fais-moi un enfant que je puisse un jour promener par la main, à côté de moi, dans Tahiti ...' (p.477). ${ }^{9}$ Avec moins de malice, mais dans la même intention, Lahontan imaginait que son sauvage avait voyagé 'en France, à la Nouvelle-York et à Québec', qu'il avait 'étudié les moeurs et la doctrine des Anglais et des Français' (p.3) et qu'il avait même lu - lui qui condamne la lecture - les fables d'Esope (p.47).

Il n'est donc pas étonnant que les Dialogues de Lahontan et le Supplément de Diderot donnent une image de la vie sauvage assez différente de celles des Nouveaux Voyages et des Mémoires d'une part, du Voyage de Bougainville d'autre part, et qu'elle soit même parfois contradictoire en elle-même. Ces déformations et ces contradictions ont été depuis longtemps relevées par la critique, qui les a reprochées à Lahontan et à 
Diderot comme des accrocs blâmables à la vérité. Nous mentionnerons seulement les plus visibles. Les sauvages d'Amérique du Nord, qui faisaient la guerre, pratiquaient l'esclavage. Sans paraître s'apercevoir qu'il y a là une grave atteinte à ses principes de liberté et d'humanité, Adario fait de l'esclavage une des commodités les plus précieuses de sa vie. Il vante à Lahontan l'agrément de la chasse aux castors où, dit-il, 'nos esclaves ont la plus grande peine', et la richesse et la gloire qu'il acquerrait en faisant la guerre comme les Hurons: 'plus on prendrait d'esclaves, moins on travaillerait; en un mot, vous seriez aussi heureux que nous' (p. 58 et 56). Bien qu'un jeune Huron ait le droit d'épouser l'esclave de sa mère, qui n'est pas faite autrement qu' une femme libre (p.98), c'est une bassesse pour une fille que de se donner à un esclave (p.24) - alors que les filles sont 'maîtresses de leur corps' jusqu'au mariage -, et il vaut mieux fuir devant l'ennemi et courir jour et nuit sans manger plutôt que de tomber en esclavage (p.59). Pourtant les Mémoires nous apprenaient que l'esclave était souvent adopté par la nation qui l'avait pris, et même épousé par une femme restée veuve; les autres prisonniers, il est vrai, étaient ou fusillés, ou brûlés sur des bûchers après des tortures atroces: adoption et massacre sont également éliminés des Dialogues, où ne subsiste que le contraste entre les sauvages libres et disposant d'esclaves et les Européens civilisés, prétendûment libres, en réalité esclaves du bas de l'échelle sociale jusqu'en haut. De même à Tahiti, si l'on en croit Bougainville, 'la distinction des rangs est fort marquée, et la disproportion cruelle. Les rois et les grands ont droit de vie ou de mort sur leurs esclaves et valets; je serais même tenté de croire qu'ils ont aussi ce droit barbare sur les gens du peuple' (Voyage autour du monde, II,3, éd. Jacques Proust, 1982, p.267). Diderot ne dit rien de l'esclavage ni des cruelles distinctions, mais Orou révèle que les Tahitiens ont des ennemis, par lesquels ils ont sans doute été vaincus, puisqu'ils ont 'à payer une redevance en hommes à un voisin oppresseur' (p.500); leurs terres manquent de bras, leurs armées de soldats, des épidémies les déciment. Où sont donc la santé, la fraternité, la liberté, le bonheur de vivre, l'innocence qu'exaltait le vieillard? Des discordances analogues apparaissent à propos des moeurs sexuelles. Lahontan raconte dans ses Nouveaux Voyages (p.161) une offre de politesse sauvage, fort semblable à la politesse tahitienne, que lui avait faite le chef $d$ 'un peuple inconnu près de la source de la Rivière longue. ${ }^{10}$ Ce trait de moeurs, que les Hurons ne connaissaient sans doute pas, est absent des Dialogues, mais Lahontan y insiste beaucoup plus que dans les Mémoires sur la liberté sexuelle des sauvages, eit ose quelques propos égrillards qui auraient été très déplacés dans un ouvrage ethnologique. ${ }^{11}$ Diderot, lui, non seulement explique ce qu'est la politesse tahitienne, mais prête aux Tahitiens une pratique de l'inceste paternel, 
maternel, fraternel dont Bougainville ne fait aucune mention. Quant à la religion des sauvages, nos deux philosophes, pour l'opposer à la religion des civilisés, la réduisent à un déisme rationnel ou à un athéisme naturaliste, en ignorant les sacrifices humains, les tabous, les fêtiches que les explorateurs comme Bougainville et les missionnaires avaient signalés, et les cérémonies que Lahontan avait lui-même décrites dans les Mémoires (p.125-129). D'autres anomalies ont été encore relevées chez Lahontan: les enfants des Hurons sont libres dans le choix de leur conjoint, mais la fille d'Adario va se marier 'malgré lui'; selon les Mémoires (p.133-134), les filles qui sont 'maîtresses de leur corps' se font avorter, parce qu'elles ne trouveraient jamais à se marier si elles avaient un enfant; mais dans les Dialogues (p.92), Adario donne raison à Lahontan qui condamne l'avortement comme un crime; selon les Mémoires (p.110) les sauvages 'écoutent les vieillards comme des oracles,' mais une fille que cite Adario dans les Dialogues (p.89) se révolte contre un vieillard 'en lui reprochant qu'un homme d'esprit ne devait jamais s'exposer à donner des conseils aux personnes dont il pourrait en recevoir.' Les deux écrivains ont visé le même but: donner de la vie sauvage une image paradoxale, mais non absurde. Il est paradoxal que les filles se donnent à qui leur plaît et avortent pour pouvoir se marier. Il est paradoxal qu'une fille supplie qu' on lui fasse un enfant, pour qu'elle puisse se marier. Le paradoxe est frappant, il donne à réfléchir, parce qu'il fait ressortir une vérité en s'écartant du fait. Il est d'ailleurs probable que Diderot a inventé le paradoxe de la fille qui ne peut se marier que si elle est mère, en retournant le paradoxe énoncé par Lahontan de la fille qui avorte pour pouvoir se marier. Le paradoxe de Lahontan était dans (ce qu'il croyait) la vérité des sauvages: il l'atténuait pour rendre possible le dialogue et acceptable au lecteur l'identification avec Adario; Diderot, plus profond et plus ironique, imagine un paradoxe sans réalité, mais conforme à la nature, dont les désirs sont légitimes et dont les fins sont la procréation. La sexualité est le domaine où le préjugé de la civilisation, où l'effet dénaturant de la civilisation sont le plus sensibles: l'acte sexuel est entouré de pudeur, il est lié au sentiment en dehors duquel il est obscène, et le sentiment est sanctionné par l'institution qui veut le rendre définitif.

L'écart entre ce que nous pourrions appeler la vérité documentaire (celle que Lahontan montre dans ses deux autres écrits, celle dont Bougainville est le témoin) et la représentation philosophique est mieux maîtrisé par Diderot que par Lahontan. Diderot, en jouant de cet écart, fait comprendre au lecteur, avant que $B$ ne le lui dise expressérnent, que la vie sauvage réelle est un exemple et non pas un modèle. Lahontan s'y essaie, mais avec moins d'habileté. L'image de la vie sauvage dans les Dialogues est flottante, on pourrait en dire ce que le professeur Jonas 
Conrad Schramm disait, sans doute à tort, de la philosophie des Canadiens telle que la formulait Lahontan, qu'elle est 'balbutiante. ${ }^{\prime 2}$ Elle plie les faits selon les besoins de l'argumentation. Ainsi, quand il s'agit de prouver les heureux effets d'une vie naturelle, Adario dit des vieillards qu'ils gardent leur activité jusqu'à plus de cent ans, et, presque aussitôt après, qu'ils sont comme morts après quatrevingts ans et demeurent 'dans une si molle indolence qu'ils ne sortent de leur demeure que lorsque le feu s'y met' (p. 76 et 77): c'est qu'alors il reproche aux Européens dans la force de l'âge d'être aussi paresseux que les octogénaires de son pays.

Une contradiction qui n'est qu'apparente va nous découvrir le principe de sa philosophie, nullement balbutiante. 'Nous croyons (déclare Adario dès le premier entretien) que le Grand Esprit nous a pourvus d'une raison capable de discerner le bien d'avec le mal, comme le ciel d'avec la terre, afin que nous suivions exactement les véritables règles de la justice et de la sagesse [. . .]. Que la portée de notre esprit ne pouvant s'étendre un pouce au-dessus de la superficie de la terre, nous ne devons pas le gâter ni le corrompre en essayant de pénétrer les choses invisibles et improbables.' 'Opposition flagrante,' qui 'annule la validité logique des propos,' pense Claude Rigault. Mais Adario, c'est-à-dire Lahontan, l'auteur qui le fait parler, ajoute un peu plus loin: 'Nous ne savons pas si ce que nous croyons être un mal selon les hommes l'est aussi selon Dieu,' et s'explique quelques pages plus loin encore: 'Pour moi, je crois que les hommes sont dans l'impuissance de connaître ce que le Grand Esprit demande d'eux [. . .]; Crois-moi, les affaires de l'autre monde sont bien différentes de celles-ci' (p.2-3 et 32). Nous avons en effet assez de discernement pour reconnaître ce qui nous est bon ou mauvais et pour nous bien conduire dans cette vie; le vrai, à la différence du faux, se manifeste par une évidence claire et distincte; mais 'parce qu'il n'y a que Dieu seul qui sache parfaitement toutes choses, il est besoin que nous nous contentions de saisir celles qui sont le plus à notre usage.' Cette dernière phrase n'est pas de Lahontan, elle est de Descartes écrivant le 16 septembre 1645 à la princesse Elisabeth de Bohème. Lahontan est cartésien, des dizaines de passages le prouvent. Il fait appel à la raison universelle; le Huron Adario, 'sauvage de bon sens' - et le bon sens est 'la chose du monde la mieux partagée' - , ne veut croire 'que ce [qu'il] entend et que la raison [lui] enseigne'; 'croire, c'est être persuadé; être persuadé, c'est voir de ses propres yeux une chose ou la reconnaître par des preuves claires et solides' (p. 4 et 9). Comme d'autres disciples de Descartes, comme Malebranche, Fontenelle ou Voltaire, Lahontan fait confiance à la raison dans les limites de l'humanité; il se méfie d'elle quand elle ne s'aide plus de l'expéri- 
ence et quand elle se met au service des passions. ${ }^{13}$ Héritier de Montaigne aussi, Lahontan est déjà un philosophe du XVIIIe siècle; rationaliste, sceptique, il veut faire son bonheur sur la terre en respectant la nature et la raison, sans se tourmenter pour une autre vie: ainsi il peut accéder à la 'tranquillité de l'âme.' L'expression faisait, comme l'on sait, le titre d'un traité de Sénèque, De Tranquillitate animi. 'Les Hurons ne connaissent d'autre félicité que la tranquillité d'âme et la liberté,' ils ne sont pas 'esclaves de leurs passions,' leurs âmes sont 'exemptes de passion', 'chacun est maître de soi-même' (p.61-62). Les Mémoires disaient seulement (p.99) que les sauvages savaient 'passer la vie dans une tranquillité parfaite' et demeurer dans 'une parfaite indolence sur toute chose' (p.110). Les termes sont voisins, l'idée est toute différente: à l'indolence naturelle, le sauvage des Dialogues substitue l'ataraxie philosophique, et une grande idée de la sagesse antique apparaît sous la fabulation américaine. Sans doute, les Mémoires attestent que Lahontan a demandé aux sauvages leur avis sur la religion chrétienne et sur le gouvernement des Français: il menait alors son enquête d'officier colonial, jugeant son esprit 'trop superficiel pour philosopher sur l'origine, la croyance, les moeurs et les manières de tant de sauvages' (Nouveaux Voyages, Lettre XVI, p.180). C'est quand il s'écarte d'eux qu'il commence à philosopher.

La pensée de Diderot a été trop souvent commentée pour que nous l'analysions à notre tour. La critique de la civilisation porte sur les mêmes points chez les deux philosophes: ils dénoncent l'absurdité des croyances chrétiennes, l'intolérance religieuse, l'arbitraire du pouvoir politique et sa confusion avec le pouvoir religieux, l'aliénation de la liberté par l'argent, l'oppression résultant du droit abusif de propriété, surtout quand il est exercé sur des personnes humaines, la vanité agressive de chaque individu contre tous, l'illusion sur la possibilité d'engagements éternels, le carcan du mariage à vie, l'arbitraire et l'artifice des moeurs 'honnêtes,' de la pudeur; dans le monde civilisé, tout est égoïsme, hypocrisie, mensonge, servitude et malheur. Du point de vue qui est le nôtre ici, les différences sont secondaires, bien qu'elles soient fortes: sur la nature, sur la matière, sur l'esprit humain et sa faculté de connaissance, les principes de Diderot ne sont pas ceux de Lahontan, si Lahontan s'en est même donné; l'analyse des bases de la société est plus méthodique chez Diderot; son, idéal moral, tel qu'il découle de la psychologie sensualiste, est l'activité et non l'ataraxie; la différence la plus importante est dans le résultat de la confrontation entre le sauvage et le civilisé. Chez Lahontan, elle aboutit à une double impossibilité: 'Fais-toi Huron,' dit le sauvage à son interlocuteur qui n'en a ni l'envie ni la faculté, tandis qu'Adario se promet bien de ne jamais revenir en Europe. Chez Diderot, la 
conclusion est le plan de conduite que le civilisé se propose de suivre dans sa cité.

Mais les Dialogues et le Supplément ont fondamentalement en commun l'usage que le philosophe y fait de l'exemple sauvage. Dans la réalité, si le sauvage peut recevoir du civilisé quelque bien matériel, grâce par exemple au commerce dont Adario fait l'éloge, il n'a rien à apprendre de lui, ni en bien, ni en mal. 'Vices et vertus, tout est également dans la nature,' a déclaré Diderot (p.507) et d'ailleurs, remarque Lahontan, 'il faut faire une grande différence entre les divers peuples du Canada' (Mémoires, p.103). Le civilisé est très conscient de la barbarie, des difficultés et des servitudes dans lesquelles vivent les sauvages, ces 'canailles' comme Lahontan encore, l'officier français en service au Canada, évidemment, et non l'interlocuteur d'Adario (Nouveaux Voyages, p.161), appelle les Gnacsitares. En définitive, l'état sauvage n'est ni meilleur ni pire que l'état civilisé. Mais l'idée de nature, sur laquelle s'élève toute la condamnation du civilisé par le sauvage, le civilisé seul peut la concevoir; la liberté, la tranquillité d'âme, la solidarité, l'altruisme, ces valeurs ne sont telles que pour lui; c'est la civilisation qui en crée le besoin en même temps que la notion, et elles prennent corps à condition que, sortant de la subjectivité et du réseau de relations par lesquels il est déterminé, le civilisé projette hors de lui-même un témoin qui leur prête une réalité objective, qui dévoile par opposition l'étrangeté et la monstruosité de ce qui paraissait normal et habituel. La fable du sauvage produit ce témoin, capable par excellence de désigner les maux de la civilisation, ce modèle par rapport auquel le civilisé se mesure, qui n'a de sens que pour lui, et qu'il dégage d'un exemple concret - Tahiti ou le pays des Hurons - en le façonnant sur les déficiences de sa propre condition. Nous l'avons dit, ces textes sont pleins d'indices qui révèlent volontairement le trucage.

Est-ce à dire que le sauvage fournisse à Lahontan et à Diderot seulement un point de vue, un décentrement, ou, pour parler un jargon moderne, une instance de discours? Non. S'ils ont pu transformer l'exemple en modèle, $c^{\prime}$ est parce qu'ils l'ont étudié sérieusement, $c^{\prime}$ est parce qu'ils reconnaissaient en eux-mêmes les capacités de souffrance et les aptitudes au bonheur des sauvages, une parenté de nature, qu'elle vînt - pour le déiste Lahontan - de la raison divine créatrice de l'humanité, ou - pour le moniste Diderot - d'une identité d'organisation physique. Frères différents, mais frères dont ils respectaient la différence, et dont la ressemblance les aidait à être mieux civilisés.

HENRI COULET

Université d'Aix-en-Provence 


\section{Notes}

1 Voir M. Roelens: 'Lahontan dans l'Encyclopédie et ses suites,' dans Recherches nouvelles sur quelques écrivains des Lumières, sous la direction de J. Proust, Genève, 1972.

2 La remarque est d'Ernst Rettich, dans son ouvrage de 1976, Der nackte Philosoph.

3 Réal Ouellet, 'L'oeuvre de Lahontan: une subversion du discours historique canonique,' Scritti sulla Nouvelle-France nel Seicento, Quaderni del Seicento francese, VI, Bari-Paris, 1984; 'La fin du voyage. Hasard et parodie chez Lahontan,' Voyages en Nouvelle-France, Etudes françaises, 22/2, automne 1986; Claude Rigault, 'Discontinuités et séries au début du XVIIIe siècle: la pratique historique de Lahontan,' L'Histoire au XVIIIe siècle, Actes du colloque d'Aix-enProvence des 1, 2 et 3 mai 1978, Aix-en-Provence, 1980.

4 Nous citons les textes de Lahontan d'après les éditions originales parues en 1703 chez les frères Lhonoré, à La Haye, et le Supplément au voyage de Bougainville d'après le texte publié par Paul Vernière dans le volume d'Oeuvres philosophiques de Diderot, collection des Classiques Garnier, Paris, s.d.

5 Dans la lettre XXI des Nouveaux Voyages et dans la préface des Dialogues (qui est en réalité une préface aux Nouveaux Voyages et aux Mémoires), Lahontan s'en prenait aux ministres d'Etat et nommément à Pontchartrain. Il parlait alors en son propre nom et sans déguisement.

6 L'ironie de Lahontan auteur quand il fait parler Lahontan interlocuteur va jusqu'au sarcasme: 'L'amour des Européennes est charmant, elles sont constantes et fidèles juqu'à la mort; lorsqu'elles ont la faiblesse d'accorder à leurs amants la dernière faveur, c'est plutôt en vertu de leur mérite intérieur qu'extérieur, et toujours moins par le désir de se contenter elles-mêmes que de donner des preuves sensibles d'amour à leurs amants' (p.101).

7 Voir les textes de La Condamine et de Bachaumont cités par L. Sozzi dans son article: 'La favola, la legge, l'attesa. Riforme e utopia nel Supplément di Diderot,' Il Confronto letterario, III, 5, mai 1986, p.3-57 (les citations sont aux p. 12-14).

8 L'éloquent avertissement du vieillard aux Tahitiens deviendra un avertissement aux Hottentots dans un texte rédigé par Diderot pour l'Histoire philosophique des établissements dans les deux Indes, de Raynal (Pensées détachées, publiées par Gianluigi Goggi, Siena, 1975).

9 Quelques vers plus haut (v.302), Virgile comparait Didon à une bacchante, 'Thyias'; la fille d'Orou s'appelle Thia: Diderot s'amuse.

10. La réalité de cette expédition et l'existence de la Rivière longue ont été mises en doute par plusieurs commentateurs de Lahontan.

11. Sur le jeune Français qui embrasse six fois la nuit les sauvagesses, tandis que le jeune Huron n'en fait que la moitié; sur la fille qui n'est pas pucelle parce qu'elle crie et qu'elle jure de l'être; sur les sauvagesses dont 'les unes aiment un homme bien fait, quoiqu'il ait je ne sais quoi de petit en lui; d'autres aiment un mal bâti pourvu qu'elles y trouvent je ne sais quoi de grand [etc.]' (p. 83,93,97).

12. Le 10 mai 1707 le professeur Schramm prit pour thème de sa leçon inaugurale à l'Université Julia Carolina de Helmstadt 'La philosophie balbutiante des Canadiens.' Réal Ouellet a reproduit ce texte très curieux dans sa brochure Sur Lahontan, comptes rendus et critiques (1702-1711), Québec, 1983, p.73-97. Comme 
beaucoup d'autres, Schramm ne fait aucune différence entre les Mémoires et les Dialogues.

13. Le sauvage qui parle de la guerre, dans les Mémoires (p.174-175), pense que 'la raison des hommes est le plus grand instrument de leur malheur' en les élevant au-dessus de la pure animalité et en leur donnant les moyens de se détruire les uns les autres. On croit déjà entendre le mot de Rousseau 'L'homme qui médite est un animal dépravé.' Adario déplore que l'homme sache lire et écrire et qu'il ait inventé les sciences; mais, en vrai cartésien, il admire les mathématiques. 